\title{
Observation of changes in model-based insulin sensitivity during haemodialysis transitions for critically ill patients
}

\author{
Ummu K. Jamaludin* Paul D. Docherty* J. Geoffrey Chase* \\ Aaron Le Compte* Geoffrey M. Shaw** \\ *Centre of Bioengineering, University of Canterbury, New Zealand \\ (e-mail: ummu.jamaludin@pg.canterbury.ac.nz). \\ **Department of Intensive Care Medicine, Christchurch Hospital, New Zealand
}

\begin{abstract}
Most critically ill patients exhibit a myriad of symptoms with irregular glycaemic regulation and renal failure contributing to a large proportion of mortality and morbidity. Hence, the effect of dialysis on glycaemic regulation should be of interest to clinicians as it is a common therapy addressing renal failure.

In this investigation, we measure transient changes in model-based insulin sensitivity during the commencement and end of dialysis periods in 51 critically ill patients with acute renal failure (ARF). The clinically validated model-based insulin sensitivity $\left(S_{I}\right)$ metric is a lumped parameter, which, in this case, can account for variance in insulin pharmacokinetics and production, as well as the efficiency of insulin mediated glucose uptake. Apparent $S_{I}$ is expected to be higher during renal failure as insulin will not be cleared as fast as the model assumed. Thus, it is hypothesized given model assumptions on steady, population levels of renal insulin clearance, that dialysis will cause a drop in model-based $S_{I}$, and vice versa. This study is the first to investigate the effect of dialysis on insulin action of critically ill patients.

This investigation found a significant reduction in model-based $S_{I}$ after the commencement of dialysis, but an insignificant change when dialysis was stopped. As dialysis was considered to have little effect on true insulin sensitivity and the effect on insulin production would have been contrary to the observed behavior, it was concluded that commencing dialysis had a significant effect in increasing insulin clearance over the cohort. This effect was not reciprocated immediately following dialysis due to a slower return to complete renal failure following treatment.
\end{abstract}

Keywords: insulin sensitivity, acute renal failure, haemodialysis, Specialized Relative Insulin Nutrition Titration (SPRINT), dialysis transition, critically ill patients, tight glycaemic control.

\section{INTRODUCTION}

Acute renal failure (ARF) is a common complication for critically ill patients. Approximately $36 \%$ of critically ill patients are diagnosed with ARF (De Mendonca et al., 2000, Uchino et al., 2005, Metnitz et al., 2002). ARF can cause costly morbidities, with a significant proportion of patients progressing to end-stage renal disease requiring dialysis (Star, 1998). Several epidemiological studies have shown an increase in morbidity and mortality following the development of ARF (Bentley, 2011).

The increasing incidence of ICU-related ARF may be explained by several factors, including a rising incidence of sepsis (Bagshaw et al., 2007), major surgery (especially cardiothoracic), nephrotoxic medications, and chronic medical conditions (history of chronic disease, congestive heart failure, and diabetes mellitus) (Hoste et al., 2003). In critically ill patients with renal failure, both uremia and dialysis can complicate glycaemic control by affecting secretion, insulin clearance, gluconeogenesis (Van Den
Berghe et al., 2001), and peripheral tissue sensitivity of insulin (Shrishrimal et al., 2009). Hence, the time course of the important renal failure on metabolic behaviour in the critically ill is unknown.

However, the net effect of haemodialysis on glycaemic regulation and insulin sensitivity in a critically ill cohort is unknown.

This study uses dense clinical data and a model-based analysis to observe changes in a clinically validated modelbased $S_{I}$ metric at haemodialysis transitions in a cohort of critically ill patients. Specifically, we hypothesized that model-based $S_{I}$ would fall during dialysis due to enhanced insulin clearance not seen in the model, and would thus rise when dialysis is stopped. These changes in $S_{I}$ would provide a unique view on insulin action in the population of critically ill patients with ARF. 


\section{METHODS}

\subsection{Study Cohort}

Blood glucose $(G)$, nutrition and insulin $(I)$ data from 51 critically ill patients with ARF were used in this study. Haemodialysis (HD) with polysulfone (PS) dialyzer was adapted to these patients where they were part of the SPRINT (Chase et al., 2008) study. These patients were haemodialysed three times weekly for at least 4 hour sessions at Intensive Care Unit (ICU) in Christchurch Hospital, New Zealand.

Study inclusion required a minimum of 5 hours of patient data before dialysis, followed by at least 6 hours of dialysis, and then at least 5 hours after dialysis. The clinical details of this cohort, including baseline variables, are summarized in Table 1.The APACHE III diagnosis for these patients can be divided into 5 main groups: Sepsis (19/51), Cardiovascular (10/51), Trauma (9/51), Diabetes (7/51), and Respiratory $(6 / 51)$. Full details on the patient cohort and SPRINT study can be found in (Chase et al., 2008).

Table 1. Patient Details Summary (median [IQR])

\begin{tabular}{|c|c|c|c|}
\hline Group & $\begin{array}{c}\text { Gender } \\
\mathrm{M} / \mathrm{F}\end{array}$ & $\begin{array}{c}\text { Age } \\
\text { (years) }\end{array}$ & $\begin{array}{c}\text { APACHE II } \\
\text { Score }\end{array}$ \\
\hline \hline Non-diabetic & $34 / 10$ & $65[46-73]$ & $24[19-28]$ \\
\hline Diabetic & $5 / 2$ & $73[53-73]$ & $34[22-36]$ \\
\hline
\end{tabular}

\subsection{Identification of Model-Based $S_{I}$}

Model-based $S_{I}$ is identified hourly by fitting $G$ measurements with estimated endogenous insulin secretion using the ICING model (Lin et al., 2011). An integral-based method (Docherty et al., 2009) and clinical data are used to identify a patient-specific stepwise $S_{I}$ profile with 1-hour resolution. The model is defined:

$$
\begin{gathered}
\dot{G}(t)=-p_{G} G(t)-S_{I} G(t) \frac{Q(t)}{1+\alpha_{G} Q(t)} \\
+\frac{P(t)+E G P-C N S}{V_{G}} \\
\dot{Q}(t)=n_{I}(I(t)-Q(t))-n_{C} \frac{Q(t)}{1+\alpha_{G} Q(t)} \\
\dot{I}(t)=n_{K} I(t)-n_{L} \frac{I(t)}{1+\alpha_{I} I(t)}-n_{I}(I(t)-Q(t)) \\
\quad+\frac{u_{e x}(t)}{V_{I}}+\left(1-x_{L}\right) \frac{u_{e n}(G)}{V_{I}} \\
\dot{P}_{1}(t)=-d_{1} P_{1}+D(t) \\
\dot{P}_{2}(t)=-\min \left(d_{2} P_{2}, P_{\max }\right)+d_{1} P_{1} \\
P(t)=\min \left(d_{2} P_{2}, P_{\max }\right)+P N(t)
\end{gathered}
$$

where: $G\left(\mathrm{mmol} . \mathrm{L}^{-1}\right)$ denotes the glucose above an equilibrium level, $I$ and $Q$ are plasma and interstitial insulin, respectively (mU.L $\left.\mathrm{L}^{-1}\right)$, exogenous insulin is $u_{e x}\left(\mathrm{mU} \cdot \mathrm{min}^{-1}\right), n_{I}$ $\left(\mathrm{min}^{-1}\right)$ defines the diffusion constant of insulin between compartments, and $n_{C}\left(\mathrm{~min}^{-1}\right)$ is the cellular insulin clearance rate from interstitium. Patient endogenous glucose removal and insulin sensitivity are $p_{G}\left(\mathrm{~min}^{-1}\right)$ and $S_{I}\left(\mathrm{~L} \cdot \mathrm{mU}^{-1} \cdot \mathrm{min}^{-1}\right)$, respectively, glucose and insulin distribution volume are $V_{G}$ (L) and $V_{I}(\mathrm{~L})$, Michaelis-Menten functions are used to portray saturations, with $\alpha_{I}\left(\mathrm{~L} . \mathrm{mU}^{-1}\right)$ dictating the saturation of plasma insulin clearance, and $\alpha_{G}\left(\mathrm{~L} \cdot \mathrm{mU}^{-1}\right)$ for saturation of insulin-mediated glucose removal, $n_{K}\left(\mathrm{~min}^{-1}\right)$ and $n_{L}\left(\mathrm{~min}^{-1}\right)$ are the renal and hepatic insulin clearance rates, respectively, $x_{L}$ is the first pass hepatic clearance ratio. $P_{1}$ and $P_{2}(\mathrm{mmol})$ represent the glucose in the stomach and gut, respectively, $P$ is the glucose appearance in plasma $\left(\mathrm{mmol} \cdot \mathrm{min}^{-1}\right)$ from enteral and parenteral nutrition. The parameters $d_{1}$ and $d_{2}$ are used to describe the rate of glucose transport through the enteral route into the bloodstream. The rate of transport from $P_{2}$ is limited to the maximal gut glucose flux, $P_{\max }$, and also $D$ represents the glucose input from enteral infusion $\left(\mathrm{mmol} . \mathrm{min}^{-1}\right)$. The parenteral (intravenous) glucose input rate is represented as $P N\left(\mathrm{mmol} . \mathrm{min}^{-1}\right)$. The endogenous insulin production $u_{e n}\left(\mathrm{mU} \cdot \mathrm{min}^{-1}\right)$ is defined:

$$
\begin{aligned}
& u_{e n}(G) \\
& =\left\{\begin{aligned}
u_{\min }, & u_{\min }>k_{1} G(t)-k_{2} \\
k_{1} G(t)+k_{2}, & u_{\min } \leq k_{1} G(t)-k_{2} \leq u_{\max } \\
u_{\max }, & u_{\max }<k_{1} G(t)-k_{2}
\end{aligned}\right.
\end{aligned}
$$

\begin{tabular}{|c|c|c|c|}
\hline \multicolumn{4}{|c|}{ Model parameter values } \\
\hline$p_{G}$ & \multicolumn{2}{|c|}{0.006} & $\min ^{-1}$ \\
\hline$\alpha_{G}$ & \multicolumn{2}{|c|}{0.0154} & L.mU $\mathrm{m}^{-1}$ \\
\hline$E G P$ & \multicolumn{2}{|c|}{1.16} & mmol.min ${ }^{-1}$ \\
\hline CNS & \multicolumn{2}{|c|}{0.3} & mmol.min ${ }^{-1}$ \\
\hline$V_{G}$ & \multicolumn{2}{|c|}{13.3} & $\mathrm{~L}$ \\
\hline$n_{I}$ & \multicolumn{2}{|c|}{0.006} & $\min ^{-1}$ \\
\hline$n_{C}$ & \multicolumn{2}{|c|}{0.006} & $\min ^{-1}$ \\
\hline$n_{K}$ & \multicolumn{2}{|c|}{0.0542} & $\min ^{-1}$ \\
\hline$n_{L}$ & \multicolumn{2}{|c|}{0.1578} & $\min ^{-1}$ \\
\hline$\alpha_{I}$ & \multicolumn{2}{|c|}{0.0017} & L.mU $\mathrm{mU}^{-1}$ \\
\hline$V_{I}$ & \multicolumn{2}{|c|}{4.0} & $\mathrm{~L}$ \\
\hline$x_{L}$ & \multicolumn{2}{|c|}{0.67} & \\
\hline$d_{1}$ & \multicolumn{2}{|c|}{0.0347} & $\min ^{-1}$ \\
\hline$d_{2}$ & \multicolumn{2}{|c|}{0.0069} & $\min ^{-1}$ \\
\hline$P_{\max }$ & \multicolumn{2}{|c|}{6.11} & mmol.min ${ }^{-1}$ \\
\hline$u_{\min }$ & \multicolumn{2}{|c|}{16.7} & mU.min $\min ^{-1}$ \\
\hline$u_{\max }$ & \multicolumn{2}{|c|}{266.7} & mU.min $\min ^{-1}$ \\
\hline \multirow{3}{*}{$k_{l}$} & NGT & 14.9 & \multirow{3}{*}{$\mathrm{mU} \cdot$ L. $\mathrm{mmol}^{-1} \cdot \mathrm{min}^{-1}$} \\
\hline & T2DM & 4.9 & \\
\hline & T1DM & 0.0 & \\
\hline \multirow{3}{*}{$k_{2}$} & NGT & -49.9 & \multirow{3}{*}{ mU.min ${ }^{-1}$} \\
\hline & T2DM & -27.4 & \\
\hline & T1DM & 16.7 & \\
\hline
\end{tabular}

where: $u_{\min }\left(\mathrm{mU} \cdot \mathrm{min}^{-1}\right)$ and $u_{\max }\left(\mathrm{mU} \cdot \mathrm{min}^{-1}\right)$ are the minimum and maximum pancreatic secretion rate, while $k_{1}$ and $k_{2}$ are defined by normal glucose tolerance (NGT), Type I diabetes mellitus (T1DM), and Type 2 diabetes mellitus (T2DM).

Table 2. Parameters and kinetic values based on diabetic status 

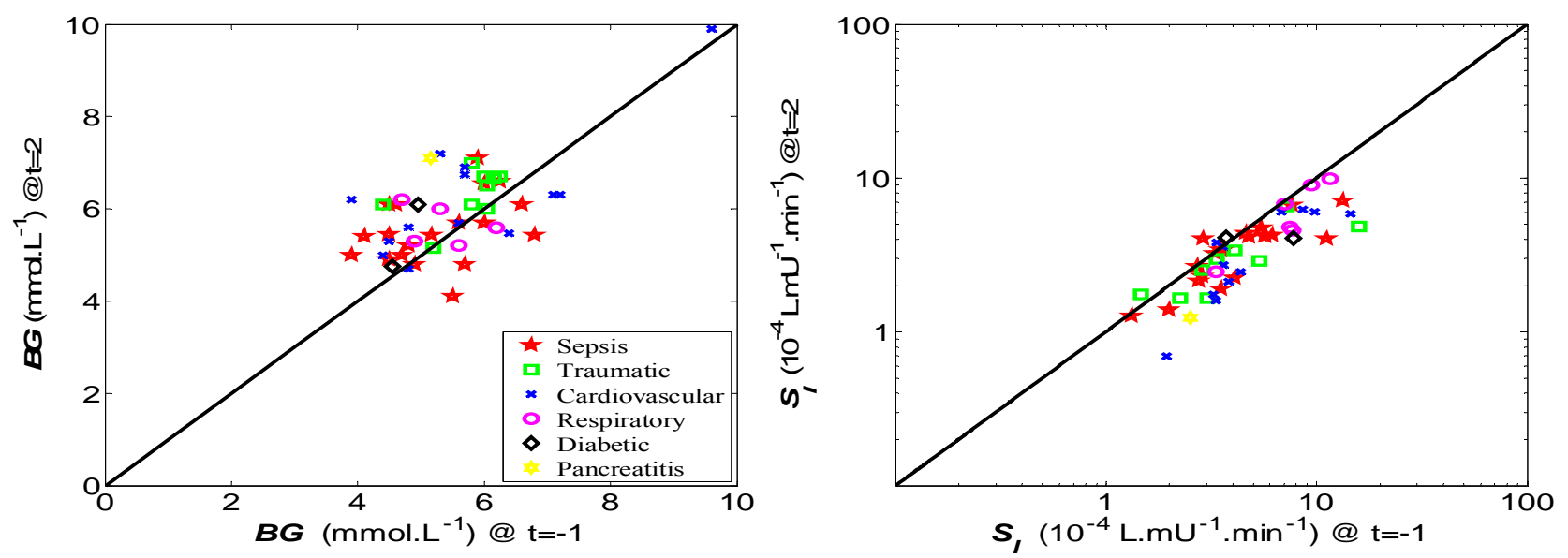

Figure 1. $G$ (left) and $S_{I}$ (right) distribution over the OFF/ON dialysis transition between $* t=-1$ and $2(\mathrm{~N}=51)$

Table 2 defines all parameter values used. Note that renal clearance is set for a relatively normal level equivalent to glomerular filtration rate (GFR).

\subsection{Data Analysis}

The proportional difference in model identified $S_{I}\left(\Delta S_{I}\right)$ was calculated as:

$\Delta S_{I}=\frac{S_{I(\text { after })}-S_{I(\text { before })}}{\text { mean }\left(S_{I \text { (before })}, S_{I(\text { after })}\right)}$

Blood glucose changes, $\Delta G$ were calculated in a similar manner to $\Delta S_{I}$ to access any change in glycaemia that could affect results.

This analysis uses a 2-hour moving average to reduce the effect of measurement error, noise, and the influence of transient effects caused by the cohort-constant assumption of these model terms. $S_{I}$ profiles are identified over periods starting 3 hours before a dialysis commencement until 4 hours after the dialysis ends. This limit ensures full settling of patient responses after transitions. Results are illustrated via $G$ (linear-linear) and $S_{I}(\log -\log )$ plots. The logarithm plot is applied to illustrate $S_{I}\left(10^{-4}\right)$ distribution at the OFF/ON transition. Wilcoxon rank sum tests are used to measure the significance of any shift in $S_{I}$ over the cohort at each transition.

\section{RESULTS}

Figure 1 shows the distribution of $G$ and $S_{I}$ before and during dialysis over the $\mathrm{OFF} / \mathrm{ON}$ transition at the commencement of dialysis. Note $G$ is stable, but $S_{I}$ is slightly biased. Figures 2 and 3 show $\Delta S_{I}$ over time for the OFF/ON and ON/OFF dialysis transitions, which clearly showing the shift in $S_{I}$.

Table 3 (left) summarizes $\Delta S_{I}$ over the OFF/ON transition for the cohort. A decrease of $S_{I}$ was observed after dialysis. The change is significant $(p \leq 0.05)$ after $\mathrm{t}=1$ hours indicating an almost immediate effect. $\Delta S_{I}$ settled to a median reduction of $-29 \%$ at $\mathrm{t}=2$ hours. There were a moderate number of

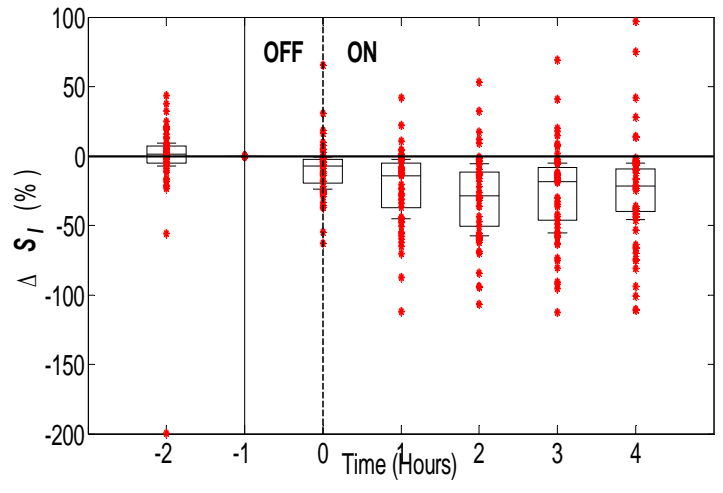

Figure 2. $\Delta S_{I}$ over $\mathrm{OFF} / \mathrm{ON}$ dialysis transition. $t=0$ indicates commencement of dialysis.



Figure 3. $\Delta S_{I}$ over ON/OFF dialysis transition. $t=0$ indicates completion of dialysis.

confounders $\left(\Delta S_{I}>0\right)$. This implies that the observation was obscured by patient variability or that the effect itself is not always observable.

$\Delta S_{I}$ after the ON/OFF dialysis transition are shown in Table 3 (right). Median $\Delta S_{I}$ was insignificant at this transition. Hence, the number of confounders was higher after the ON/OFF dialysis transition. 
Table 3. Summary of $\Delta S_{I}$ at both haemodialysis transition, OFF/ON and ON/OFF, $(\mathrm{N}=51)$

\begin{tabular}{|c|c|c|c|c|c|c|c|c|c|c|c|c|}
\hline \multirow{2}{*}{$\begin{array}{l}\text { Time } \\
*_{\mathrm{t}}\end{array}$} & \multicolumn{6}{|c|}{ OFF/ON } & \multicolumn{6}{|c|}{ ON/OFF } \\
\hline & $\mathrm{Q}_{1}$ & $\mathrm{Q}_{2}$ & $\mathrm{Q}_{3}$ & $p$-value & $S_{I}(\uparrow)$ & $\%$ & $\mathrm{Q}_{1}$ & $\mathrm{Q}_{2}$ & $\mathrm{Q}_{3}$ & $p$-value & $S_{I}(\downarrow)$ & $\%$ \\
\hline-2 & -7 & 1 & 10 & 0.9 & $(31 / 51)$ & 61 & -13 & -4 & 3 & 0.7 & $(32 / 51)$ & 63 \\
\hline-1 & 0 & 0 & 0 & 1 & $(0 / 51)$ & 0 & 0 & 0 & 0 & 1 & $(0 / 51)$ & 0 \\
\hline 0 & -24 & -7 & -1 & 0.3 & $(10 / 51)$ & 20 & -5 & 1 & 8 & 0.9 & $(24 / 51)$ & 47 \\
\hline 1 & -45 & -14 & -2 & 0.05 & $(7 / 51)$ & 14 & -7 & 2 & 15 & 0.9 & $(23 / 51)$ & 45 \\
\hline 2 & -58 & -29 & -6 & 0.02 & $(5 / 51)$ & 10 & -13 & 4 & 26 & 0.8 & $(21 / 51)$ & 41 \\
\hline 3 & -55 & -19 & -5 & 0.03 & $(9 / 51)$ & 18 & -15 & 9 & 28 & 0.7 & $(23 / 51)$ & 45 \\
\hline 4 & -46 & -22 & -5 & 0.03 & $(6 / 51)$ & 12 & -15 & 3 & 31 & 0.5 & $(24 / 51)$ & 47 \\
\hline
\end{tabular}

are 2 -hour averages rounded at the time shown, and dialysis transition

In both cases, $G$ remained effectively constant with insignificant changes $(p>0.61)$. Hence, the impact of insulin sensitivity changes at both transitions on glycaemia was quickly accounted for by the SPRINT glycaemic control protocol and there were no difference in glycaemia. Equally, there is no impact on the results due to change in glycaemia.

\section{DISCUSSION}

This study investigated changes in a clinically validated model-based $S_{I}$ metric at transitions onto and off haemodialysis in a critically ill cohort undergoing glycaemic control. Significant insulin sensitivity changes were observed at the OFF/ON dialysis transition $(p<0.05)$. This analysis shows that model-based $S_{I}$ decreased over the initial 4-hours of haemodialysis and the change was almost immediate. This result potentially indicates that dialysis significantly affected plasma insulin levels as expected, which, in turn, dictates glucose metabolism.

Glucose intolerance among critically ill patients with ARF occurred with significant inhibition of insulin secretion and a state of peripheral insulin resistance (Defronzo et al., 1978, Mak, 1995) in addition to insulin resistance from critical illness (Basi et al., 2005). Thus, a reduction of available insulin may cause hyperglycaemia to occur during the initial periods of dialysis if tight glycaemic control (TGC) is not concurrent.

A tendency for inhibited insulin secretion has also been observed in patients with severely compromised renal function (Fliser et al., 1998). The effects of insulin resistance can be exacerbated by impairment of the role of insulin in maintaining the hepatic glucose balance (Defronzo et al., 1981). Specifically, an inability of insulin to stimulate hepatic glucose uptake with decreasing insulin sensitivity was observed in acute renal failure patients (Valera Mora et al., 2003). Thus, understanding the pharmacokinetics of insulin during dialysis is important clinically.

$\Delta S_{I}$ at the $\mathrm{ON} / \mathrm{OFF}$ dialysis transition was insignificant. It is assumed that acute i.v. administration of 1,25dihydroxyvitamin $\mathrm{D}_{3},\left(1,25(\mathrm{OH})_{2} \mathrm{D}_{3}\right)$ given to ARF patients during haemodialysis may increase insulin secretion and reverse glucose intolerance (Mak, 1995). An improvement in glucose metabolism was observed after a second study period by a lower mean glucose during dialysis and more rapid disappearance rate of glucose in the immediate post-dialysis period (Alfrey et al., 1967). Also, the glucose metabolism and renal function are expected to increase gradually in postdialysis compare with pre-dialysis when toxic substances that are suspected of hindering renal function are extracted. The insulin catabolism in patients with chronic uremia is reportedly reduced. This is mainly due to the lack of functioning renal tissue accompanied by toxic suppression of both insulin secretion and degradation, mitigated by dialysis (Navalesi et al., 1975). However, it has not been conclusively demonstrated that this might happen within the ARF patients undergoing haemodialysis in critical care. In addition, longer post-dialysis duration would likely show significant change in $S_{I}$, but 10 to 15 hours of investigation post-dialysis would be impossible to segregate changes in $S_{I}$ from other issues and dysfunction of critical illness (Hampers et al., 1966). Thus, a substantial change in $S_{I}$ at $\mathrm{OFF} / \mathrm{ON}$ transition indicates a continuous and fast process of cleaning and clearing waste substances from blood. However, at the ON/OFF transition, the recovery process to regulate and normalize blood is a lot slower physiologically. Hence, the model-based $S_{I}$, after dialysis in this study may be expected to remain unchanged, as observed.

Changes in $G$ at the dialysis transitions were minimal indicating successful TGC was achieved with the SPRINT protocol (Chase et al., 2008). Almost no bias in $\Delta G$ was observed. Thus, the confounding factor plays no role. The outlier observed in Figure 1 with $G \approx 10 \mathrm{mmol} . \mathrm{L}^{-1}$.suffered from cardiovascular disease and was identified as at risk of acute renal failure and diabetes Thus, this patient's glucose control of was not fully realised in the timeframe presented here due to the complex interactions between these diseases (Sarnak et al., 2003).

The model-based $S_{I}$ is an indication of overall glucose metabolism of critically ill patients and does not necessarily reflect the precise physiology of insulin. The changes in the model-based $S_{I}$ at a cohort level are unlikely to be caused in this case by actual variance in $S_{I}$ at cellular level. In particular, there is no apparent stimulus induced by haemodialysis to affect $S_{I} . \Delta S_{I}$ was most likely affected either by renal clearance changes or/and endogenous insulin secretion resulting in an increase in apparent $S_{I}$ in the model.

The ICING model prediction of $u_{e n}$ is made in terms of glucose concentration in the absence of direct measurement. 
Hence, the effect of dialysis on $u_{e n}$ may be poorly defined by the model. However, it has been shown that insulin also suppresses insulin production (Argoud et al., 1987, Liljenquist et al., 1978) . As plasma insulin concentration is suspected to decrease during dialysis, it may also be suspected that endogenous insulin production would be increasing, at a cohort level. This would actually increase the observed modelled $S_{I}$ over time, which is contrary to the observation. Hence $u_{e n}$ dependence can be discounted as a potential reason for the shifts in modelled $S_{I}$ in this study.

Overall, it would seem that the most likely contributor to the observed changes in $S_{I}$ was the rate of insulin clearance. SPRINT reduced mortality and morbidity rates among critically ill patients with tighter control than existing, more ad-hoc clinical approaches (Chase et al., 2008). There is still potential for improvement within this model-based TGC methodology. The effect of haemodialysis on plasma insulin and the mechanism of insulin clearance among critically ill patients with ARF were shown in this study to be a factor in governing $S_{I}$, which drives model-based glycaemic control. However, further study must be undertaken to measure the effects of dialysis on the pharmacokinetics and dynamics relevant to glucose metabolism in the critically ill. A prospective cohort and clinical studies on critically ill patients with ARF may provide better context in understanding insulin kinetics during haemodialysis. Comparison from a different cohort with mixed levels of insulin resistance will also provide clearer effect of $\Delta S_{I}$, which may reveal further information in the underlying factors of specific insulin resistance.

\section{CONCLUSION}

The findings of this study show distinct changes in modelbased insulin sensitivity during haemodialysis as an observable aspect of critically ill patient physiology. However, the precise pharmacokinetics/dynamics driving this change remain ambiguous. These results justify larger cohort investigation.

\section{REFERENCES}

Alfrey, A.C., Sussman, K.E. \& Holmes, J.H. (1967). Changes in glucose and insulin metabolism induced by dialysis in patients with chronic uremia. Metabolism, 16(8), 733-40.

Argoud, G.M., Schade, D.S. \& Eaton, R.P. (1987). Insulin suppresses its own secretion in vivo. Diabetes, 36(8), 959-62.

Bagshaw, S.M., Uchino, S., Bellomo, R., Morimatsu, H., Morgera, S., Schetz, M., Tan, I., Bouman, C., Macedo, E., Gibney, N., Tolwani, A., OudemansVan Straaten, H.M., Ronco, C., Kellum, J.A., For The, B. \& Ending Supportive Therapy for the Kidney, I. (2007). Septic Acute Kidney Injury in Critically Ill Patients: Clinical Characteristics and
Outcomes. Clinical Journal of the American Society of Nephrology, 2(3), 431-9.

Basi, S., Pupim, L.B., Simmons, E.M., Sezer, M.T., Shyr, Y., Freedman, S., Chertow, G.M., Mehta, R.L., Paganini, E., Himmelfarb, J. \& Ikizler, T.A. (2005). Insulin resistance in critically ill patients with acute renal failure. American Journal of Physiology Renal Physiology, 289(2), F259-F64.

Bentley, M.L. (2011). Acute Kidney Insufficiency in the Critically Ill. Journal of Pharmacy Practice, 24(1), 61-9.

Chase, J.G., Shaw, G., Le Compte, A., Lonergan, T., Willacy, M., Wong, X.-W., Lin, J., Lotz, T., Lee, D. \& Hann, C. (2008). Implementation and evaluation of the SPRINT protocol for tight glycaemic control in critically ill patients: a clinical practice change. Critical Care, 12(2), R49.

De Mendonca, A., Vincent, J.L., Suter, P.M., Moreno, R., Dearden, N.M., Antonelli, M., Takala, J., Sprung, C. \& Cantraine, F. (2000). Acute renal failure in the ICU: risk factors and outcome evaluated by the SOFA score. Intensive Care Medicine, 26(7), 91521.

Defronzo, R.A., Alvestrand, A., Smith, D., Hendler, R., Hendler, E. \& Wahren, J. (1981). Insulin resistance in uremia. The Journal of Clinical Investigation, 67(2), 563-8.

Defronzo, R.A., Tobin, J.D., Rowe, J.W. \& Andres, R. (1978). Glucose Intolerance in Uremia: QUANTIFICATION OF PANCREATIC BETA CELL SENSITIVITY TO GLUCOSE AND TISSUE SENSITIVITY TO INSULIN. The Journal of Clinical Investigation, 62(2), 425-35.

Docherty, P.D., Chase, J.G., Lotz, T., Hann, C.E., Shaw, G.M., Berkeley, J.E., Mann, J.I. \& Mcauley, K. (2009). DISTq: An Iterative Analysis of Glucose Data for Low-Cost, Real-Time and Accurate Estimation of Insulin Sensitivity. The Open Medical Informatics Journal, 365-7.

Fliser, D., Pacini, G., Engelleiter, R., Kautzky-Willer, A., Prager, R., Franek, E. \& Ritz, E. (1998). Insulin resistance and hyperinsulinemia are already present in patients with incipient renal disease. Kidney Int, 53(5), 1343-7.

Hampers, C.L., Soeldner, J.S., Doak, P.B. \& Merrill, J.P. (1966). Effect of chronic renal failure and hemodialysis on carbohydrate metabolism. Journal of Clinical Investigation, 45, 1719-31.

Hoste, E.A.J., Lameire, N.H., Vanholder, R.C., Benoit, D.D., Decruyenaere, J.M.A. \& Colardyn, F.A. (2003). Acute Renal Failure in Patients with Sepsis in a Surgical ICU: Predictive Factors, Incidence, Comorbidity, and Outcome. Journal of the American Society of Nephrology, 14(4), 1022-30. 
Liljenquist, J.E., Horwitz, D.L., Jennings, A.S., Chiasson, J.L., Keller, U. \& Rubenstein, A.H. (1978). Inhibition of insulin secretion by exogenous insulin in normal man as demonstrated by C-peptide assay. Diabetes, 27563-70.

Lin, J., Razak, N.N., Pretty, C.G., Le Compte, A., Docherty, P., Parente, J.D., Shaw, G.M., Hann, C.E. \& Geoffrey Chase, J. (2011). A physiological Intensive Control Insulin-Nutrition-Glucose (ICING) model validated in critically ill patients. Comput Methods Programs Biomed, 102(2), 192-205.

Mak, R.H.K. (1995). Insulin Secretion and Growth Failure in Uremia. Pediatric Research, 38(3), 379-83.

Metnitz, P.G., Krenn, C.G., Steltzer, H., Lang, T., Ploder, J., Lenz, K., Le Gall, J.R. \& Druml, W. (2002). Effect of acute renal failure requiring renal replacement therapy on outcome in critically ill patients. Critical Care Medicine, 30(9), 2051-8.

Navalesi, R., Pilo, A., Lenzi, S., Donato, L., Cecchetti, P. \& Masoni, A. (1975). Insulin Metabolism in Chronic Uremia and in the Anephric State: Effect of the Dialytic Treatment. Journal of Clinical Endocrinology \& Metabolism, 40(1), 70-85.

Sarnak, M.J., Levey, A.S., Schoolwerth, A.C., Coresh, J., Culleton, B., Hamm, L.L., Mccullough, P.A., Kasiske, B.L., Kelepouris, E., Klag, M.J., Parfrey, P., Pfeffer, M., Raij, L., Spinosa, D.J. \& Wilson, P.W. (2003). Kidney Disease as a Risk Factor for Development of Cardiovascular Disease. Circulation, 108(17), 2154-69.

Shrishrimal, K., Hart, P. \& Michota, F. (2009). Managing diabetes in hemodialysis patients: Observations and recommendations. Cleveland Clinic Journal of Medicine, 76(11), 649-55.

Star, R.A. (1998). Treatment of acute renal failure. Kidney International, 54(6), 1817-31.

Uchino, S., Kellum, J.A., Bellomo, R., Doig, G.S., Morimatsu, H., Morgera, S., Schetz, M., Tan, I., Bouman, C., Macedo, E., Gibney, N., Tolwani, A., Ronco, C., Beginning \& Ending Supportive Therapy for the Kidney, I. (2005). Acute renal failure in critically ill patients: a multinational, multicenter study. JAMA : the journal of the American Medical Association, 294(7), 813-8.

Valera Mora, M.E., Scarfone, A., Calvani, M., Greco, A.V. \& Mingrone, G. (2003). Insulin Clearance in Obesity. Journal of the American College of Nutrition, 22(6), 487-93.

Van Den Berghe, G., Wouters, P., Weekers, F., Verwaest, C., Bruyninckx, F., Schetz, M., Vlasselaers, D., Ferdinande, P., Lauwers, P. \& Bouillon, R. (2001). Intensive insulin therapy in the critically ill patients. N Engl J Med, 345(19), 1359-67. 